\title{
Editorial
}

Molecular

Neuropsychiatry
Mol Neuropsychiatry 2019;5:5

DOI: $10.1159 / 000495247$
Received: November 2, 2018

Accepted: November 2, 2018

Published online: November 19, 2018

\section{OMICS Approaches to Unravel the Complexity of Psychiatric Disorders: Impact on Biomarker Discovery}

\author{
Daniel Martins-de-Souza \\ Laboratory of Neuroproteomics, University of Campinas, Campinas, Brazil
}

It is indispensable to this readership to defend the importance of better understanding psychiatric disorders from the molecular point of view. Knowledge on this regard is going to impact diagnosis, treatment, and disease monitoring.

From the mid-1990s, more specifically in the post-human genome era, a group of methodologies to screen in a large-scale manner the products of human cells and tissues have arisen. These methodologies, mostly transcriptomics, metabolomics (including lipidomics), and proteomics, have been helpful in the generation of new hypotheses and in the identification of potential biomarkers. If validated, these hypotheses and biomarkers may influ-

D.M.S. is funded by São Paulo Research Foundation (FAPESP), grants 2017/25588-1 2018/03673-0). ence the way psychiatric patients are diagnosed and treated, which will contribute to move psychiatry into the era of precision medicine.

Since its foundation in 2013, the Molecular Psychiatry Association has been promoting annual meetings in the USA, bringing together major experts in the field, which include several of the "omics" references. Some of these gathered in several symposia across the years and are contributing to this special issue entitled "OMICS approaches to unravel the complexity psychiatric disorders: impact on biomarker discovery" with original data as well as comprehensive reviews. The next pages are filled with pivotal knowledge, obtained in human samples and preclinical models, which brings new ideas to the field. These are potentially impactful in the search for a personalized and more precise type of medicine, so much needed in psychiatry.

Daniel Martins-de-Souza, Campinas, Brazil 